New texts in electromagnetism

I THOROUGHLY enjoyed reading J. Crangle's The Magnetic Properties of Solids (Arnold: London, hardback $£ 10$; paperback 14.95 ). It is written in an easy confident style which appealed to me and left me feeling that my understanding of the subject had been decidedly improved. The appearance of the book is reasonably pleasing too, with clear mathematical layout and good diagram locations. The unregistered right margin is only a minor irritant and has presumably helped to keep the price down.

This book is one of a series edited by Professor Bryan R. Coles under the general title The Structures and Properties of Solids. It is aimed at the "senior undergraduate studying physics in a British university" and to a lesser extent at other undergraduates, postgraduates and practising scientists. The level seems to me to be absolutely right for the specialist or optional courses which are often offered in the final year after a basic solid-state physics course. There are generous numbers of diagrams, many displaying experimental data (not always referenced-for example, figures 3.7, 4.13 and 6.13) which support the text. There are no problem sets, although this is perhaps not a serious defect. The heart of the book is, I suppose, the central group of five chapters: "Localized Magnetism Associated with the Ion Cores", "Magnetism Associated with Band Electrons", "Antiferromagnetism, Ferrimagnetism and Helimagnetism", "Magnetic Interactions and Hyperfine Fields", and "Domain Magnetism". There is also a helpful introductory chapter on basics and units (the SI system is used consistently, with c.g.s. conversions also given in brackets) and two sensible final chapters, one on experimental techniques (extremely valuable) and the other on practical applications.

I have to report that I was completely out of sympathy with the second book, R. C. Cross's Electricity and Magnetism (Longman: Sidney and Harlow, UK, paperback $£ 3$ ). Again it is one of a series, under the editorship of J. L. A. Francey, and it was written "for students taking a first course in physics at university or college". Its level seems to me to hover unhappily between an advanced level at school and that of a proper electromagnetism course for undergraduates (at a British university). In my experience of lecturing on electromagnetic theory to first-year undergraduates, students become restive if they suspect that their schoolwork is being wheeled out again. It can be done briefly, but then no textbook is needed. So I am puzzled about Cross's intentions. It is clear from what he says and from internal evidence that the book is not intended to be a physics degree-level course. To give an example, the treatment of electromagnetic waves, though interesting and physical, is rather brief and avoids heavy vector manipulation by not presenting the wave equation at all. This in my view disqualifies the book as a main recommendation for a full electromagnetism course. But then are there any universities who can spare the time for two full courses on electromagnetism, one at the level of this book and a second at degree level?

I also found as I read the book a number of detailed points which made me unhappy right from the beginning. In the hydrogen atom, the electron does not spend most of its time moving around the surface of a sphere of radius $5.3 \times 10^{-11} \mathrm{~m}$ (p7). $F=q_{1} q_{2} / 4 \pi \varepsilon_{0} r^{2}$ does not give the force between charged objects (a common student fallacy) unless those objects are small compared with their separation (p21). The argument (on p36) that electric field lines outside a conductor must be perpendicular to the surface because

\section{Electrical properties of metals}

Electrical Properties of Metals and Alloys. By J. S. Dugdale. Pp. 292. (Edward Arnold: London, 1977.) Hardback $£ 10.50$; paperback $£ 4.95$.

APART from their mechanical properties, the electrical properties of metals are surely those which are most widely exploited - and what wonderful properties they are! The electrical conductivity, the Hall effect, thermoelectricity-familiarity should not blind us to the remarkable nature of these phenomena. Their widespread applications as well as their intrinsic interest demand a reasonable degree of understanding by any physical scientist. Unfortunately, once one ventures beyond the classical Drude treatment, the theory of electrons in metals can be come very complicated, and the physics which underlies electrical conductivity and its associated properties can become lost in an assembly of matrix elements.

Professor Dugdale does not lose sight of the physics in the present carefully written volume. He takes great care to explain the physical ideas that underly the various phenomena, not only in the introductory chapters but also in the detailed exposition which follows. $\mathrm{He}$ uses simple arguments with the minimum of mathematical sophistication to explain the dynamics of electrons in metals and the various scattering mechanisms to which they are prone. The Boltzmann equation, scattering from static imperfections and phonons, the Born approxi- otherwise electrons would move inside the conductor, is not convincing without more discussion. An electric kettle operated at $0.045 \mathrm{~W}$ would never boil (p72) although admittedly for thermal rather than electrical reasons. On p156 it is remarked that students are often bothered by the fact that there may be electric fields, at points where $B=0$, which are induced by fluxes changing elsewhere. Those students are unlikely to be helped by the bare statement that "this is indeed a remarkable feat of nature" coupled to a rather doubtful analogy.

The book is pleasantly laid out and reasonably priced. It contains quite a large number of problems, generally helpful diagrams, and basic equations (not numbered, presumably deliberately) which are as light as possible on calculus and vectors.

D. S. Betts

D. S. Betts is Lecturer in Experimental Physics at the University of Sussex, UK. mations, phase shift analysis and the Friedel sum rule, are dealt with gently but firmly.

In later sections more complicated phenomena are discussed. The Kondo effect, always a tricky item, is particularly well explained and there are good sections on phonon drag and Mattheissen's rule. The final chapters cover the electrical properties of the transition metals and of alloys. The level should not cause much difficulty to any reasonably competent second-year undergraduate, although it is unlikely that any undergraduate would wish to study in such detail all the topics covered.

It is inevitable that a reliance on fairly simple mathematics necessitates more wordy explanations and some readers, particularly those with a theoretical bent, might find the pace a little slow, but this is a small price to pay for the clarity of explanation which is evident throughout the book. It is certainly one to be recommended as supplementary reading for any introductory solid-state physics course.

This book is one of a series, Structure and Properties of Solids, and in my experience the quality of individual volumes in a series can be very variable. In the present instance this is not so. Each of the volumes I have read has been of a very high standard and the editor of the series, Professor Bryan Coles, is to be commended on his selection of authors and on the control which he has apparently been able to maintain over them.

H. M. Rosenberg

H. M. Rosenberg is Reader in Physics at the Clarendon Laboratory, University of Oxford, $U K$. 